\title{
Photon energy absorption buildup factors of gaseous mixtures used in radiation detectors
}

\author{
V.P. SINGH ${ }^{1,2}$, N.M. BADIGER ${ }^{1}$
}

(Manuscript received 2 February 2012, accepted 15 May 2012)

ABSTRACT Gamma-ray energy absorption buildup factors of gaseous mixtures; neon (95\%) + argon $(5 \%)$, argon $(95 \%)+$ acetylene $(5 \%)$, argon $(95 \%)+$ methane $(5 \%)$, argon $(95 \%)+$ carbon dioxide $(5 \%)$, methane $(70 \%)$ + pentane $(30 \%)$ and argon $(90 \%)+$ methane $(\mathbf{1 0 \%})$ were studied by Geometrical Progression (G-P) fitting for the photon energy range $0.015-15 \mathrm{MeV}$. It was found that the equivalent atomic number, $Z_{\text {eq }}$ of the gaseous mixtures sharply reduces after $1 \mathrm{MeV}$ photon energy. The $Z_{\mathrm{eq}}$ for the mixture of methane $(70 \%)+$ pentane $(30 \%)$ is the minimum, whereas the maximum is for argon $(95 \%)+$ carbon dioxide $(5 \%)$ for the photon energies under investigation. The Energy Absorption Buildup Factor (EABF) for methane (70\%) + pentane $(30 \%)$ was found to be the highest among all the selected gaseous mixtures. The chemical composition of the gaseous mixtures has an impact on the EABF values for photon energy and penetration depth. The investigation of the EABF is useful for selection of gaseous mixtures in design consideration of gaseous radiation detectors for gamma radiation.

Keywords: equivalent atomic number / gamma / EABF / G-P fitting / gaseous / detector / reactor / protection / absorption / Compton scattering

\section{Introduction}

Gamma-ray energy absorption and the exposure buildup factor in the air medium has been established for the infinite medium. The air is one of the most important media for interaction of ionizing radiation for measurement of gamma radiation. Other than air, many other gases and their mixture/combinations are used for gamma radiation detection as an active volume of the detectors. Radiation measuring instruments such as the ionization chamber, proportional counter and Geiger Muller counter are commonly used for natural as well as artificial radiation. The radiation emitted from natural radioactivity is of limited photon energy, whereas gamma photon energy ranges from $0.10-10 \mathrm{MeV}$ during reactor operation and even higher energy in accelerators. Gamma radiation sources in the reactors are delayed gamma, prompt gamma, captured gamma and gamma photons emitted

\footnotetext{
Department of Physics, Karnatak University, Dharwad 580003, India.

Health Physics Section, Kaiga Atomic Power Station-3\&4, NPCIL, Karwar, Karnatak 581400, India,

e-mail: kudphyvps@rediffmail.com.
} 
due to activation (example: ${ }^{7} \mathrm{~N},{ }^{8} \mathrm{O},{ }^{11} \mathrm{Na},{ }^{18} \mathrm{Ar},{ }^{25} \mathrm{Mn},{ }^{27} \mathrm{Co},{ }^{28} \mathrm{Ni},{ }^{36} \mathrm{Kr},{ }^{37} \mathrm{Rb}$, ${ }^{40} \mathrm{Zr},{ }^{41} \mathrm{Nb},{ }^{42} \mathrm{Mo},{ }^{44} \mathrm{Ru},{ }^{54} \mathrm{Xe},{ }^{51} \mathrm{Sb},{ }^{55} \mathrm{Cs},{ }^{56} \mathrm{Ba}$, etc.). In low photon energy from a few $\mathrm{keV}$ to $\mathrm{MeV}$, photoelectric absorption and Compton scattering are the dominant interaction processes, whereas pair-production becomes significant at high photon energies. The gamma-ray photon energy absorption and exposure buildup factor for air, water, lead, concretes, boron and other combinations have been evaluated (Raza and Avila, 2005). It has been reported that conventional radiation monitors underestimate the personal dose equivalent in high-energy bremsstrahlung photon radiation fields near electron storage rings (Haridas et al., 2006). Since interaction of gamma-ray photons with the medium is energy, thickness and elements dependent, therefore the buildup factors in gaseous medium of the detectors may also be photon energy-dependent. The response of the radiation detector to high-energy gamma photons is not similar compared with low-energy gamma photons (Allard et al., 1992); therefore, the study of buildup of gamma photons in the gaseous medium is extremely important in the design of the detector and mixture selection. The application of various types of gases and mixtures of the radiation detectors also cause buildup of gamma photon energy, which needs to be studied. Effective atomic numbers, $Z_{\text {eff }}$, effective electron densities, $N_{\text {eff }}$ and tissue equivalence of some gases and gaseous mixtures of radiation detectors have been studied (Singh and Badiger, 2011, 2012a, 2012b).

The interaction of gamma-ray photons with the medium/material degrades their original energy and causes a buildup in the medium, giving rise to secondary radiation. The buildup of gamma-ray photons is estimated by the "buildup factor", a dimensionless multiplication factor which corrects the response of uncollided photon beams. The buildup is defined as the ratio of the total value of a specified radiation quantity at any point to the contribution to that value from radiation reaching the point without having undergone a collision. There are two types of buildup factors: (a) the absorbed or deposited energy in the interacting materials and detector response function is that of absorption in the interacting medium; (b) the exposure buildup factor, in which the quality of interest in the exposure and detector response function is that of absorption in air (Harima, 1993). The buildup factors have been computed by various codes such as PALLAS (Takeuchi and Tanaka, 1984, 1985, 1986), ADJMON-I (Simmons, 1973; Chilton et al., 1980), ASFIT (Gopinath and Samthanam, 1971) and EGS4 (Nelson et al., 1985).

The compilation of buildup factors was reported in gamma-ray attenuation coefficients \& buildup factors for engineering materials, ANSI/ANS-6.4.3-1991 standard, by the American Nuclear Society (ANSI, 1991), which presents evaluated gamma-ray elemental attenuation coefficients and single-material buildup factors for selected engineering materials for use in shielding calculations of structures in nuclear power plants and other nuclear facilities. The data cover the energy range 
0.015-15 MeV and up to 40 mean free paths ( $\mathrm{mfp}$ ). These data are intended to be standard reference data for use in radiation analyses employing point-kernel methods. The ANSI/ANS-6.4.3-1991 standards have been withdrawn from the American Nuclear Society but presently no reference data are available for buildup factors; therefore, these data have been taken as a reference in our study. The buildup factors in ANSI/ANS-6.4.3-1991 are for 23 nucleides for atomic numbers; $Z=4$ to $92\left({ }^{4} \mathrm{Be},{ }^{5} \mathrm{~B},{ }^{6} \mathrm{C},{ }^{7} \mathrm{~N},{ }^{8} \mathrm{O},{ }^{11} \mathrm{Na},{ }^{12} \mathrm{Mg},{ }^{13} \mathrm{Al},{ }^{14} \mathrm{Si},{ }^{15} \mathrm{P},{ }^{16} \mathrm{~S},{ }^{18} \mathrm{Ar},{ }^{19} \mathrm{~K},{ }^{20} \mathrm{Ca},{ }^{26} \mathrm{Fe}\right.$, $\left.{ }^{29} \mathrm{Cu},{ }^{42} \mathrm{Mo},{ }^{50} \mathrm{Sn},{ }^{57} \mathrm{La},{ }^{64} \mathrm{Gd},{ }^{74} \mathrm{~W},{ }^{82} \mathrm{~Pb},{ }^{92} \mathrm{U}\right)$. In the present work, we have taken standard gaseous mixtures (Knoll, 2000) for calculation of the energy absorption buildup factor (EABF) in the photon energy range $0.015-15 \mathrm{MeV}$ up to $20 \mathrm{mfp}$ or penetration depth by geometrical progression (G-P) fitting formulae developed by Harima (1993). The penetration depth of $20 \mathrm{mfp}$ was chosen because deeper is not practically possible for the detector. The G-P fitting formula is known to be excellent within the estimated error $<5 \%$ analyzed for various materials. The buildup factors of the ANSI/ANS-6.4.3-1991 standard can be calculated by other methods such as invariant embedding (Akinao, 2002; Sakamoto and Tanaka, 1988; Shimizu, 2002; Shimizu et al., 2004). The variations in the EABF with photon energy, penetration depth and their chemical compositions have also been investigat ed by G-P fitting for polymers and tissue substitute materials (Murat and Yuksel, 2011), human tissue (Murat and Yuksel, 2011), concrete shielding (Singh and Badiger, 2012a, 2012b) and dosimetric materials (Manohara et al., 2010), etc. and established the best available methodology.

Various types of radiation detectors; Geiger counter, proportional counter and ionization chambers are used for gamma radiation measurement. The EABF of the gaseous mixtures used in the radiation detectors is not found in the literature at present. Therefore, we took some different combinations of gases as neon (95\%) $+\operatorname{argon}(5 \%)$, argon $(95 \%)+$ acetylene $(5 \%)$, argon $(95 \%)+$ methane $(5 \%)$, argon $(95 \%)+$ carbon dioxide $(5 \%)$, methane $(70 \%)+$ pentane $(30 \%)$ and argon $(90 \%)+$ methane $(10 \%)$ to study the EABF of the radiation detectors comprehensively. The study can also be further extended for other combinations of the gases. The results of our investigation are very useful for understanding the behavior of the gaseous medium of detectors for amount of deposition of photon energy, required attenuation length and chemical composition dependence. The detailed analysis of the photon interaction in the gaseous medium proves its suitability for radiation monitoring and correlation with the operational quantity for radiological protection of workers from ionizing radiation. The energy of gamma photons covers the range 0.015 to $15 \mathrm{MeV}$ for $20 \mathrm{mfp}$, sufficient thickness of the gaseous medium to understand the phenomenon of interaction inside the detector. The gas pressure of the detector cavity was not considered during the investigation, although our results would also be useful for other analysis. 


\section{Computational work}

The buildup factors and the G-P fitting parameters are calculated by a method of interpolation from the equivalent atomic number, $Z_{e q}$, of the gaseous mixture. The computational work of these parameters is completed in three steps as follows:

- calculation of equivalent atomic number, $Z_{e q}$,

- calculation of G-P fitting parameters,

- calculation of buildup factors.

The $Z_{e q}$, is a parameter which describes the composite material properties in terms of equivalent elements similar to atomic numbers for a single element, and hence represents one atomic number instead of the various atomic numbers of the elements. Since the interaction process of gamma-ray photons with gaseous mixture is by photoelectric absorption, Compton scattering and pair-production is energy-dependent therefore $Z_{e q}$ for each interaction varies according to the energy of the photons. However, the buildup of photons in the gaseous medium is essentially due to multiple scattering events, so that the $Z_{e q}$ is derived from the Compton scattering interaction process.

The $Z_{e q}$ for individual gaseous mixtures is estimated by the ratio of $(\mu / \rho)_{\text {Compton }} /(\mu / \rho)_{\text {Total }}$, at a specific photon energy with the corresponding element at the same energy. Thus, first the Compton partial mass attenuation coefficient, $(\mu / \rho)_{\text {Compton }}$, and the total mass attenuation coefficient, $(\mu / \rho)_{\text {Total }}$, are obtained for elements of atomic numbers $Z=4$ to 40 for the chosen gaseous mixtures in the photon energy region 0.0015 to $15 \mathrm{MeV}$ using the WinXCom computer program developed by Gerward (Gelward et al., 2001, 2004), which was earlier developed by Hubbell (Berger and Hubbell, 1987/1999).

The interpolation of $Z_{e q}$ is employed by the formula (Harima, 1983; Maron, 1987):

$$
Z_{e q}=\frac{Z_{1}\left(\log R_{2}-\log R_{1}\right)+Z_{2}\left(\log R-\log R_{1}\right)}{\log R_{2}-\log R_{1}}
$$

where $Z_{1}$ and $Z_{2}$ are the atomic numbers of the element corresponding to the ratios $R_{1}$ and $R_{2}$, respectively. $R$ is the ratio $(\mu / \rho)_{\text {Compton }} /(\mu / \rho)_{\text {Total }}$ at specific energy and the ratio $(\mu / \rho)_{\text {Compton }} /(\mu / \rho)_{\text {Total }}$ for the $Z_{e q}$ lies between two successive ratios of the elements such that $R_{1}<R<R_{2}$. The $Z_{e q}$ values of the different selected gaseous mixtures are shown in Figure 1, which shows that the $Z_{e q}$ values are approximately constant up to $1 \mathrm{MeV}$ photon energy and afterward begin decreasing.

The G-P fitting parameters are calculated in a similar fashion to the interpolation procedure for the $Z_{e q}$. The G-P fitting parameters for the gaseous 


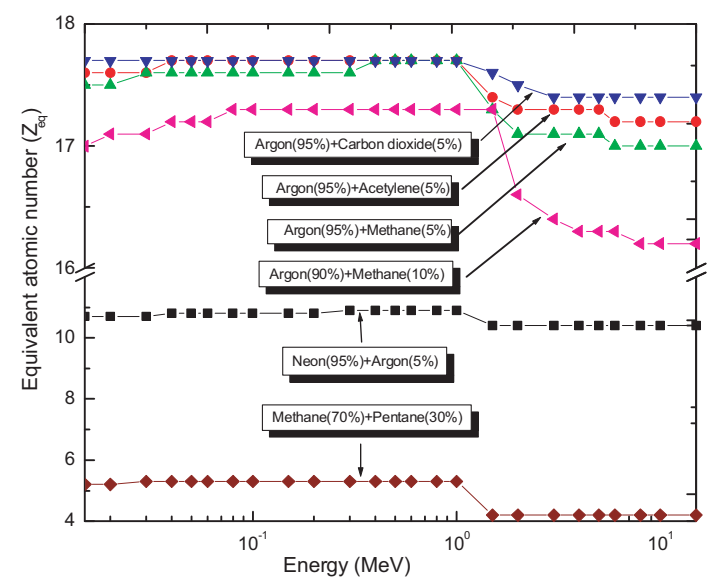

Figure 1 - Equivalent atomic number vs. photon energy.

mixtures were interpolated using standard ANSI/ANS-6.4.3-1991 standard elemental data:

$$
C=\frac{C_{1}\left(\log Z_{2-} \log Z_{e q}\right)+C_{2}\left(\log Z_{e q}-\log Z_{1}\right)}{\log Z_{2}-\log Z_{1}}
$$

where $C_{1}$ and $C_{2}$ are the values of the G-P fitting parameters corresponding to the atomic numbers of $Z_{1}$ and $Z_{2}$, respectively, at a given photon energy.

The third and final step for buildup factors is estimation of the fitting parameters $\left(b, c, a, X_{k}\right.$ and $\left.d\right)$ in the photon range of $0.015-15 \mathrm{MeV}$ by the equations (Harima et al., 1986):

$$
\begin{aligned}
& B(E, X)=1+\frac{b-1}{K-1}\left(K^{x}-1\right) \text { for } K \neq 1 \\
& B(E, X)=1+(b-1) X \text { for } K=1
\end{aligned}
$$

where

$$
\begin{gathered}
K(E, X)=c X^{a}+d \frac{\tan h\left(X / X_{K}-2\right)-\tan h(-2)}{1-\tanh (-2)} \\
\text { for penetration depth }(X) \leq 40 \mathrm{mfp}
\end{gathered}
$$

where $X$ is the source-detector distance for the medium in terms of mfp and $b$, the value of the exposure buildup factor at $1 \mathrm{mfp} . K(E, X)$ is the dose multiplicative 
factor, and $b, c, a, X_{K}$ and $d$ are computed G-P fitting parameters that depend on the attenuating medium (chemical compositions or $Z_{e q}$ ) and photon energy. The EABF parameters of the selected gaseous mixtures are given in Tables I to III.

\section{TABLE I}

G-P fitting parameters for the energy absorption buildup factor of neon $(95 \%)+\operatorname{argon}(5 \%)$ and argon $(95 \%)+$ acetylene $(5 \%)$ for the photon energy range $0.015-15 \mathrm{MeV}$.

\begin{tabular}{|c|c|c|c|c|c|c|c|c|c|c|}
\hline \multirow{2}{*}{$\begin{array}{l}\text { Energy } \\
(\mathbf{M e V})\end{array}$} & \multicolumn{5}{|c|}{ neon $(95 \%)+\operatorname{argon}(5 \%)$} & \multicolumn{5}{|c|}{ argon $(95 \%)+$ acetylene $(5 \%)$} \\
\hline & $b$ & $c$ & $a$ & $X_{k}$ & $d$ & $b$ & $c$ & $a$ & $X_{k}$ & $d$ \\
\hline 0.015 & 1.06 & 0.41 & 0.20 & 11.60 & -0.092 & 1.010 & 0.456 & 0.173 & 25.854 & -0.261 \\
\hline 0.02 & 1.14 & 0.42 & 0.20 & 14.54 & -0.109 & 1.025 & 0.435 & 0.172 & 30.230 & -0.340 \\
\hline 0.03 & 1.46 & 0.49 & 0.18 & 14.45 & -0.091 & 1.087 & 0.351 & 0.246 & 13.525 & -0.145 \\
\hline 0.04 & 1.95 & 0.65 & 0.11 & 15.96 & -0.056 & 1.194 & 0.398 & 0.211 & 14.423 & -0.116 \\
\hline 0.05 & 2.65 & 0.78 & 0.08 & 14.87 & -0.057 & 1.359 & 0.429 & 0.204 & 14.382 & -0.118 \\
\hline 0.06 & 3.27 & 0.96 & 0.02 & 14.34 & -0.025 & 1.555 & 0.520 & 0.158 & 15.392 & -0.086 \\
\hline 0.08 & 4.22 & 1.26 & -0.04 & 11.78 & 0.010 & 2.194 & 0.556 & 0.163 & 13.447 & -0.098 \\
\hline 0.1 & 4.52 & 1.48 & -0.08 & 13.38 & 0.029 & 2.786 & 0.720 & 0.100 & 13.345 & -0.073 \\
\hline 0.15 & 4.04 & 1.75 & -0.13 & 13.57 & 0.050 & 3.599 & 1.031 & 0.008 & 13.382 & -0.028 \\
\hline 0.2 & 3.60 & 1.80 & -0.13 & 13.79 & 0.081 & 3.611 & 1.220 & -0.031 & 13.289 & -0.012 \\
\hline 0.3 & 3.02 & 1.78 & -0.13 & 13.88 & 0.071 & 3.160 & 1.392 & -0.066 & 20.565 & 0.013 \\
\hline 0.4 & 2.72 & 1.71 & -0.12 & 14.18 & 0.044 & 2.831 & 1.433 & -0.075 & 16.732 & 0.016 \\
\hline 0.5 & 2.54 & 1.64 & -0.11 & 14.25 & 0.041 & 2.613 & 1.432 & -0.076 & 16.929 & 0.018 \\
\hline 0.6 & 2.40 & 1.57 & -0.10 & 14.55 & 0.037 & 2.454 & 1.416 & -0.075 & 16.905 & 0.019 \\
\hline 0.8 & 2.23 & 1.47 & -0.09 & 14.84 & 0.033 & 2.249 & 1.378 & -0.072 & 15.629 & 0.021 \\
\hline 1 & 2.11 & 1.39 & -0.08 & 14.83 & 0.028 & 2.120 & 1.334 & -0.066 & 15.028 & 0.021 \\
\hline 1.5 & 1.94 & 1.25 & -0.05 & 14.28 & 0.021 & 1.942 & 1.225 & -0.047 & 15.001 & 0.015 \\
\hline 2 & 1.84 & 1.16 & -0.03 & 14.73 & 0.013 & 1.834 & 1.151 & -0.031 & 15.673 & 0.008 \\
\hline 3 & 1.71 & 1.05 & -0.01 & 11.32 & -0.001 & 1.692 & 1.054 & -0.008 & 12.546 & -0.005 \\
\hline 4 & 1.62 & 0.98 & 0.01 & 13.12 & 0.008 & 1.592 & 0.990 & 0.011 & 12.749 & -0.018 \\
\hline 5 & 1.54 & 0.95 & 0.02 & 12.78 & 0.010 & 1.505 & 0.967 & 0.017 & 15.280 & -0.027 \\
\hline 6 & 1.48 & 0.92 & 0.02 & 15.80 & 0.017 & 1.440 & 0.947 & 0.025 & 13.813 & -0.032 \\
\hline 8 & 1.39 & 0.90 & 0.03 & 12.29 & 0.016 & 1.345 & 0.927 & 0.033 & 12.574 & -0.031 \\
\hline 10 & 1.33 & 0.89 & 0.04 & 13.83 & 0.018 & 1.284 & 0.910 & 0.040 & 13.735 & -0.041 \\
\hline 15 & 1.23 & 0.88 & 0.04 & 14.73 & 0.020 & 1.181 & 0.938 & 0.035 & 14.424 & -0.037 \\
\hline
\end{tabular}


PHOTON ENERGY ABSORPTION BUILDUP FACTORS OF GASEOUS MIXTURES

TABLE II

G-P fitting parameters for the energy absorption buildup factor of argon $(95 \%)+$ methane $(5 \%)$ and argon $(95 \%)+$ carbon dioxide $(5 \%)$ for the photon energy range $0.015-15 \mathrm{MeV}$.

\begin{tabular}{|c|c|c|c|c|c|c|c|c|c|c|}
\hline \multirow{2}{*}{$\begin{array}{l}\text { Energy } \\
(\mathrm{MeV})\end{array}$} & \multicolumn{5}{|c|}{$\operatorname{argon}(95 \%)+$ methane $(5 \%)$} & \multicolumn{5}{|c|}{$\operatorname{argon}(95 \%)+$ carbon dioxide $(5 \%)$} \\
\hline & $b$ & $c$ & $a$ & $X_{k}$ & $d$ & $b$ & $c$ & $a$ & $X_{k}$ & $d$ \\
\hline 0.015 & 1.011 & 0.447 & 0.179 & 25.072 & -0.256 & 1.010 & 0.428 & 0.195 & 23.347 & -0.244 \\
\hline 0.02 & 1.026 & 0.429 & 0.176 & 30.157 & -0.347 & 1.025 & 0.433 & 0.174 & 30.197 & -0.343 \\
\hline 0.03 & 1.088 & 0.353 & 0.245 & 13.539 & -0.144 & 1.086 & 0.346 & 0.250 & 13.475 & -0.149 \\
\hline 0.04 & 1.197 & 0.399 & 0.211 & 14.434 & -0.116 & 1.193 & 0.397 & 0.211 & 14.350 & -0.114 \\
\hline 0.05 & 1.363 & 0.431 & 0.203 & 14.392 & -0.117 & 1.357 & 0.415 & 0.212 & 14.287 & -0.123 \\
\hline 0.06 & 1.560 & 0.521 & 0.157 & 15.387 & -0.086 & 1.551 & 0.500 & 0.165 & 15.444 & -0.091 \\
\hline 0.08 & 2.204 & 0.559 & 0.162 & 13.438 & -0.097 & 2.188 & 0.519 & 0.179 & 13.568 & -0.104 \\
\hline 0.1 & 2.797 & 0.724 & 0.099 & 13.353 & -0.072 & 2.779 & 0.665 & 0.118 & 13.229 & -0.081 \\
\hline 0.15 & 3.606 & 1.034 & 0.008 & 13.368 & -0.027 & 3.595 & 0.960 & 0.025 & 13.646 & -0.037 \\
\hline 0.2 & 3.613 & 1.223 & -0.032 & 13.462 & -0.011 & 3.610 & 1.152 & -0.017 & 9.643 & -0.021 \\
\hline 0.3 & 3.159 & 1.394 & -0.067 & 20.449 & 0.014 & 3.160 & 1.342 & -0.058 & 23.258 & 0.010 \\
\hline 0.4 & 2.831 & 1.434 & -0.075 & 16.737 & 0.016 & 2.830 & 1.406 & -0.071 & 16.611 & 0.014 \\
\hline 0.5 & 2.612 & 1.433 & -0.077 & 16.892 & 0.018 & 2.613 & 1.402 & -0.071 & 17.869 & 0.015 \\
\hline 0.6 & 2.454 & 1.417 & -0.075 & 16.872 & 0.019 & 2.454 & 1.391 & -0.070 & 17.760 & 0.016 \\
\hline 0.8 & 2.249 & 1.378 & -0.072 & 15.622 & 0.021 & 2.249 & 1.366 & -0.069 & 15.795 & 0.019 \\
\hline 1 & 2.120 & 1.334 & -0.066 & 15.044 & 0.021 & 2.120 & 1.333 & -0.066 & 14.632 & 0.021 \\
\hline 1.5 & 1.941 & 1.226 & -0.047 & 14.984 & 0.015 & 1.942 & 1.231 & -0.048 & 14.878 & 0.016 \\
\hline 2 & 1.835 & 1.151 & -0.031 & 15.687 & 0.008 & 1.833 & 1.143 & -0.029 & 15.847 & 0.006 \\
\hline 3 & 1.692 & 1.055 & -0.008 & 12.447 & -0.004 & 1.692 & 1.064 & -0.012 & 11.214 & 0.000 \\
\hline 4 & 1.592 & 0.991 & 0.010 & 12.800 & -0.017 & 1.592 & 1.007 & 0.003 & 13.464 & -0.008 \\
\hline 5 & 1.507 & 0.966 & 0.017 & 15.184 & -0.027 & 1.504 & 0.956 & 0.019 & 13.948 & -0.022 \\
\hline 6 & 1.440 & 0.948 & 0.024 & 14.026 & -0.031 & 1.439 & 0.960 & 0.016 & 16.755 & -0.027 \\
\hline 8 & 1.346 & 0.927 & 0.032 & 12.655 & -0.030 & 1.345 & 0.936 & 0.024 & 13.712 & -0.020 \\
\hline 10 & 1.285 & 0.910 & 0.040 & 13.686 & -0.040 & 1.283 & 0.906 & 0.037 & 13.056 & -0.032 \\
\hline 15 & 1.183 & 0.936 & 0.035 & 14.394 & -0.038 & 1.180 & 0.901 & 0.042 & 14.011 & -0.041 \\
\hline
\end{tabular}


TABLE III

G-P fitting parameters for the energy absorption buildup factor of methane $(\mathbf{7 0} \%)+$ pentane $(30 \%)$ and argon $(90 \%)+$ methane $(10 \%)$ for the photon energy range $0.015-15 \mathrm{MeV}$.

\begin{tabular}{|c|c|c|c|c|c|c|c|c|c|c|}
\hline \multirow{2}{*}{$\begin{array}{c}\text { Energy } \\
(\mathrm{MeV})\end{array}$} & \multicolumn{5}{|c|}{ methane $(70 \%)+$ pentane $(30 \%)$} & \multicolumn{5}{|c|}{ argon $(90 \%)+$ methane $(10 \%)$} \\
\hline & $b$ & $c$ & $a$ & $X_{k}$ & $d$ & $b$ & $c$ & $a$ & $X_{k}$ & $d$ \\
\hline 0.015 & 1.670 & 0.643 & 0.110 & 14.882 & -0.054 & 1.012 & 0.397 & 0.219 & 20.596 & -0.225 \\
\hline 0.02 & 2.492 & 0.899 & 0.036 & 15.445 & -0.027 & 1.030 & 0.392 & 0.202 & 29.707 & -0.394 \\
\hline 0.03 & 4.454 & 1.500 & -0.088 & 14.239 & 0.035 & 1.095 & 0.362 & 0.237 & 13.631 & -0.136 \\
\hline 0.04 & 5.416 & 2.086 & -0.167 & 14.267 & 0.072 & 1.214 & 0.400 & 0.212 & 14.512 & -0.118 \\
\hline 0.05 & 5.690 & 2.370 & -0.193 & 14.674 & 0.080 & 1.389 & 0.443 & 0.197 & 14.466 & -0.113 \\
\hline 0.06 & 5.364 & 2.558 & -0.209 & 14.789 & 0.085 & 1.599 & 0.534 & 0.152 & 15.354 & -0.083 \\
\hline 0.08 & 4.652 & 2.703 & -0.220 & 14.938 & 0.085 & 2.275 & 0.578 & 0.154 & 13.375 & -0.094 \\
\hline 0.1 & 4.116 & 2.713 & -0.220 & 15.233 & 0.082 & 2.877 & 0.749 & 0.091 & 13.405 & -0.068 \\
\hline 0.15 & 3.611 & 2.449 & -0.194 & 16.500 & 0.062 & 3.653 & 1.061 & 0.001 & 13.268 & -0.024 \\
\hline 0.2 & 3.229 & 2.339 & -0.187 & 16.981 & 0.067 & 3.626 & 1.246 & -0.037 & 14.723 & -0.008 \\
\hline 0.3 & 2.746 & 2.187 & -0.181 & 15.092 & 0.071 & 3.158 & 1.410 & -0.070 & 19.604 & 0.015 \\
\hline 0.4 & 2.562 & 1.996 & -0.163 & 14.494 & 0.065 & 2.833 & 1.442 & -0.077 & 16.773 & 0.016 \\
\hline 0.5 & 2.438 & 1.842 & -0.145 & 14.998 & 0.061 & 2.610 & 1.441 & -0.078 & 16.619 & 0.019 \\
\hline 0.6 & 2.371 & 1.702 & -0.126 & 14.807 & 0.049 & 2.452 & 1.425 & -0.077 & 16.628 & 0.020 \\
\hline 0.8 & 2.190 & 1.580 & -0.112 & 14.131 & 0.048 & 2.249 & 1.382 & -0.072 & 15.575 & 0.021 \\
\hline 1 & 2.086 & 1.471 & -0.095 & 14.034 & 0.041 & 2.122 & 1.335 & -0.066 & 15.155 & 0.021 \\
\hline 1.5 & 1.933 & 1.294 & -0.064 & 13.755 & 0.029 & 1.941 & 1.226 & -0.047 & 14.977 & 0.015 \\
\hline 2 & 1.836 & 1.182 & -0.042 & 13.871 & 0.019 & 1.837 & 1.149 & -0.031 & 15.730 & 0.008 \\
\hline 3 & 1.713 & 1.059 & -0.015 & 13.147 & 0.007 & 1.691 & 1.058 & -0.009 & 12.073 & -0.003 \\
\hline 4 & 1.637 & 0.979 & 0.006 & 14.010 & -0.004 & 1.591 & 0.996 & 0.008 & 13.003 & -0.014 \\
\hline 5 & 1.574 & 0.940 & 0.015 & 13.945 & -0.008 & 1.511 & 0.963 & 0.018 & 14.804 & -0.026 \\
\hline 6 & 1.533 & 0.898 & 0.028 & 13.801 & -0.014 & 1.443 & 0.952 & 0.022 & 14.873 & -0.030 \\
\hline 8 & 1.454 & 0.867 & 0.037 & 10.838 & -0.016 & 1.350 & 0.930 & 0.030 & 12.986 & -0.027 \\
\hline 10 & 1.402 & 0.844 & 0.043 & 13.630 & -0.022 & 1.290 & 0.908 & 0.039 & 13.488 & -0.038 \\
\hline 15 & 1.310 & 0.821 & 0.050 & 13.679 & -0.026 & 1.188 & 0.925 & 0.037 & 14.273 & -0.039 \\
\hline
\end{tabular}




\section{Results and discussion}

Uncertainties in the EABF estimation by G-P fitting are one of the important aspects for suitability of the data. The buildup factors by ANSI/ANS/6.4.3-1991 and G-P fitting formulae have been verified for air and water by several investigators (Murat et al., 2011; Manohara et al., 2010). In addition, the Exposure Buildup Factor (EBF) of water has been compared for ANSI/ANS/6.4.3-1991, G-P fitting and the MCNP5 photon energy range 0.015-15 MeV and various penetration depths (Singh and Badiger, 2012a, 2012b) which is worthless to describe here. The MCNP5 results vary from those of the standard ANSI/ANS/6.4.3-1991 with a maximum deviation of $13.83 \%$ due to difference in cross-section libraries, method of solution for codes, calculation methods, standard deviation and physics assumptions for bremsstrahlung and coherent scattering (Luis, 2009). These quantitative and comparative analysis shows that our results in the present work for gamma-ray EABFs in the gaseous mixture will be within the acceptable range of uncertainties. The absolute values of maximum deviation in the EBF for water in G-P fitting are within 0.5-3\%, which are much lower compared with Berger and Taylor (Harima et al., 1986). It is found that the calculation of the buildup factor database in the present work and ANSI/ANS/6.4.3-1991 agrees for air and water within a few percentage of uncertainty analysis. Therefore, the G-P fitting method seems to reproduce the EABF with acceptable accuracy. The investigations of differences in the estimated buildup factor by the present method with standard data are in excellent agreement. This microscopic uncertainty analysis gives us confidence in our result for estimation of the EABF for mixtures of gases used in radiation detectors.

\subsection{Variation of energy absorption buildup factor with photon energy}

Variations in EABF values of gaseous mixtures with photon energy are shown in Figure 2. We found that the EABFs of the gaseous mixtures are very small at low and high photon energies, whereas they are higher at the intermediate photon energies. At low and high photon energies, photoelectric absorption and pairproduction are the dominant interaction processes which remove the photons from the gaseous medium. From Figure 2, it can be seen that the photoelectric absorption at low photon energy is significant and the EABF for high $Z_{e q}$, $\operatorname{argon}(95 \%)+$ carbon dioxide $(5 \%)$ is smaller compared with methane $(70 \%)+$ pentane $(30 \%)$. The reason is that the photoelectric absorption is directly proportional to $Z^{4-5}$ and $E^{-7 / 2}$; therefore, $Z_{e q}$ plays a vital role in the low photon energy region. The buildup factor for high $Z_{e q}$ gaseous mixtures at low photon energy $(<0.05 \mathrm{MeV})$ is observed to be negligible because of maximum removal of 

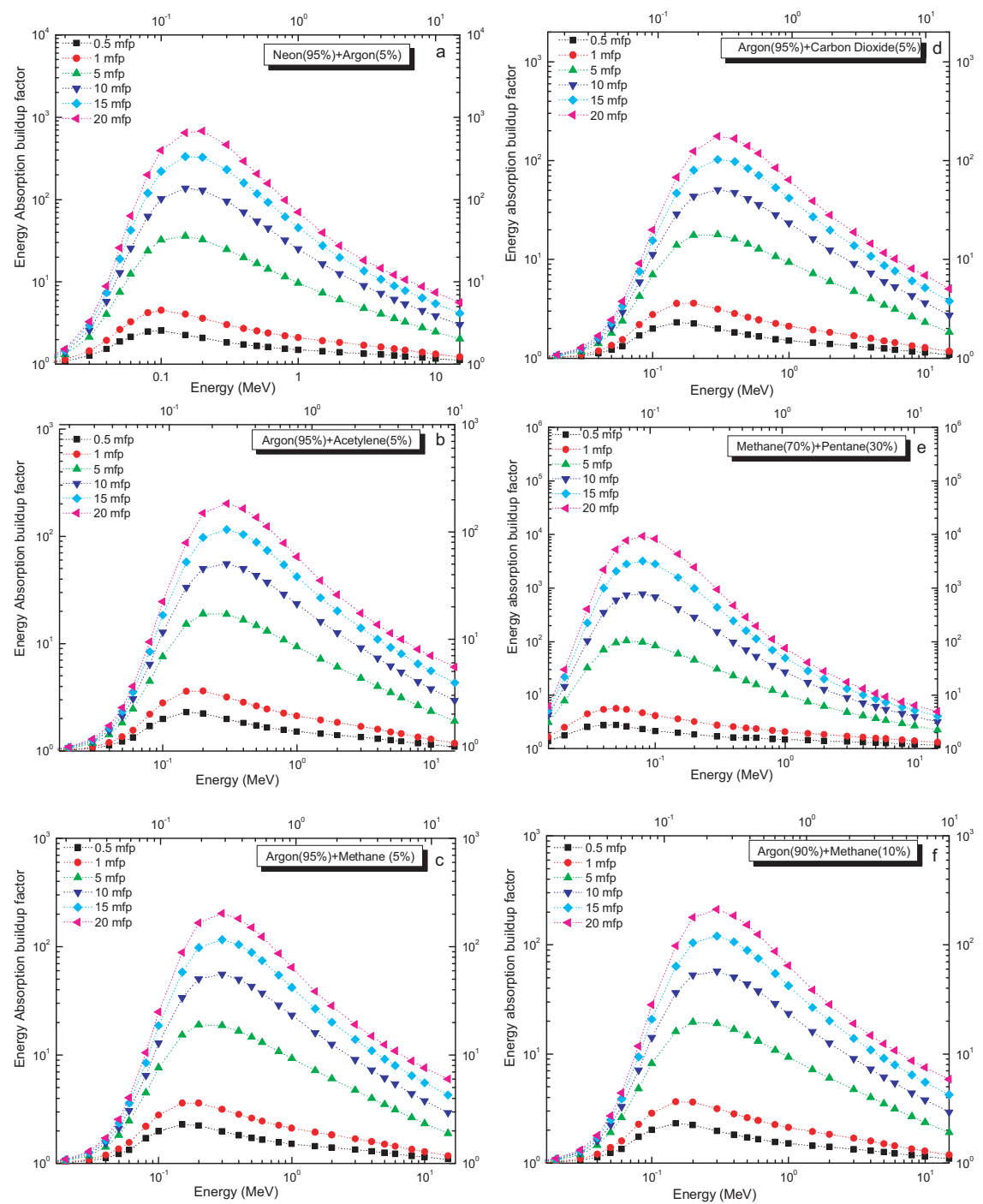

Figure 2 - Variation in energy absorption buildup factor with photon energy. 
photons from the mixtures. In the intermediate energy region, the buildup factor increases due to multiple scattering of photons by the gas molecules as the Compton scattering cross-section is of greater value as increase linearly with $Z$ and inversely proportional to photon energy $\left(E^{-1}\right)$. The energy at which the buildup factor is maximum, $E_{\max }$, shifts towards higher values as the attenuation thickness or penetration depth increases. The mixture of argon (95\%) + carbon dioxide (5\%) shows the lowest EABF values, whereas neon $(95 \%)+\operatorname{argon}(5 \%)$ and methane $(70 \%)+$ pentane $(30 \%)$ show high values.

\subsection{Variation in energy absorption buildup factor with penetration depth}

Variation in EABFs with penetration depth is shown in Figure 3. We found that the $\mathrm{EABF}$ increases with the increase in the penetration depth of the gaseous mixtures of the radiation detectors. The EABF of the selected gaseous mixture is found to be constant ( unity) at a photon energy of $0.015 \mathrm{MeV}$. The EABFs at $0.015,0.15,1.5$ and $15 \mathrm{MeV}$ photon energies are in the ran es 1.04-6.25, 1.98$4308.71,1.43-40.83$ and 1.01-6.06, respectively. The maximum buildup factor is found to be of the order of $10^{4}$ at $20 \mathrm{mfp}$ for methane $(70 \%)+$ pentane (30\%) at a photon energy of $0.15 \mathrm{MeV}$ and the minimum of the order of 1 at $0.015 \mathrm{MeV}$ for neon $(95 \%)+\operatorname{argon}(5 \%)$. It is to be noted that the EABF at a photon energy of $15 \mathrm{MeV}$ is always less than at $0.15 \mathrm{MeV}$ and $1.5 \mathrm{MeV}$, whereas it is greater than at $0.015 \mathrm{MeV}$. Although, in the case of methane $(70 \%)+$ pentane $(30 \%)$, the buildup factor is observed to be the lowest at a photon energy of $15 \mathrm{MeV}$, which may be due to having the lowest $Z_{e q}$ of gaseous mixture.

\subsection{Variation in energy absorption buildup factor with chemical compositions}

The EABF at a particular penetration depth varies with the variation in photon energy due to the chemical compositions of the gases. Figure 4 shows that the buildup factors for the mixtures of the gases increase with the increase in the penetration depth, as the EABF for methane (70\%) + pentane (30\%) goes from 3.6 at $0.5 \mathrm{mfp}$ up to the order of $10^{4}$ at $20 \mathrm{mfp}$. It is observed that the buildup factors increase with the increase in penetration depths for low $Z_{e q}$ up to $5 \mathrm{mfp}$, whereas comparatively higher $Z_{e q}$ start increasing afterward. It can be explained by the photoelectric absorption as cross-section $\tau \propto Z^{4-5} / E^{7 / 2}$ so the high $Z_{e q}$ gaseous mixture in low photon energy region remove photons which result in lower buildup factor. At a photon energy of $15 \mathrm{Mev}$, the Compton scattering crosssection is lowest; therefore, the buildup factors seem to be independent of the chemical compositions of the gaseous mixtures and have similar order values for 

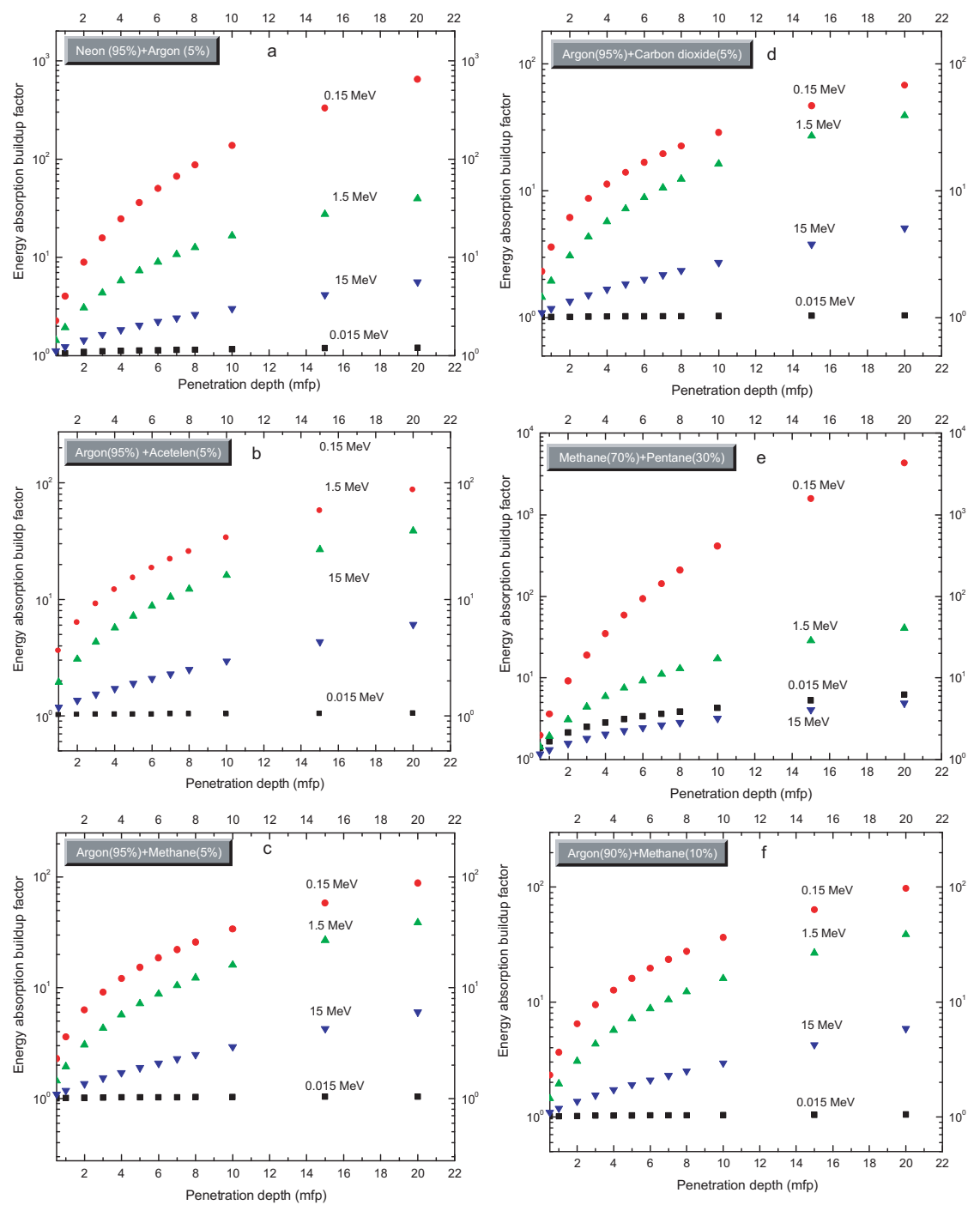

Figure 3 - Variation in energy absorption buildup factor with the penetration depth. 

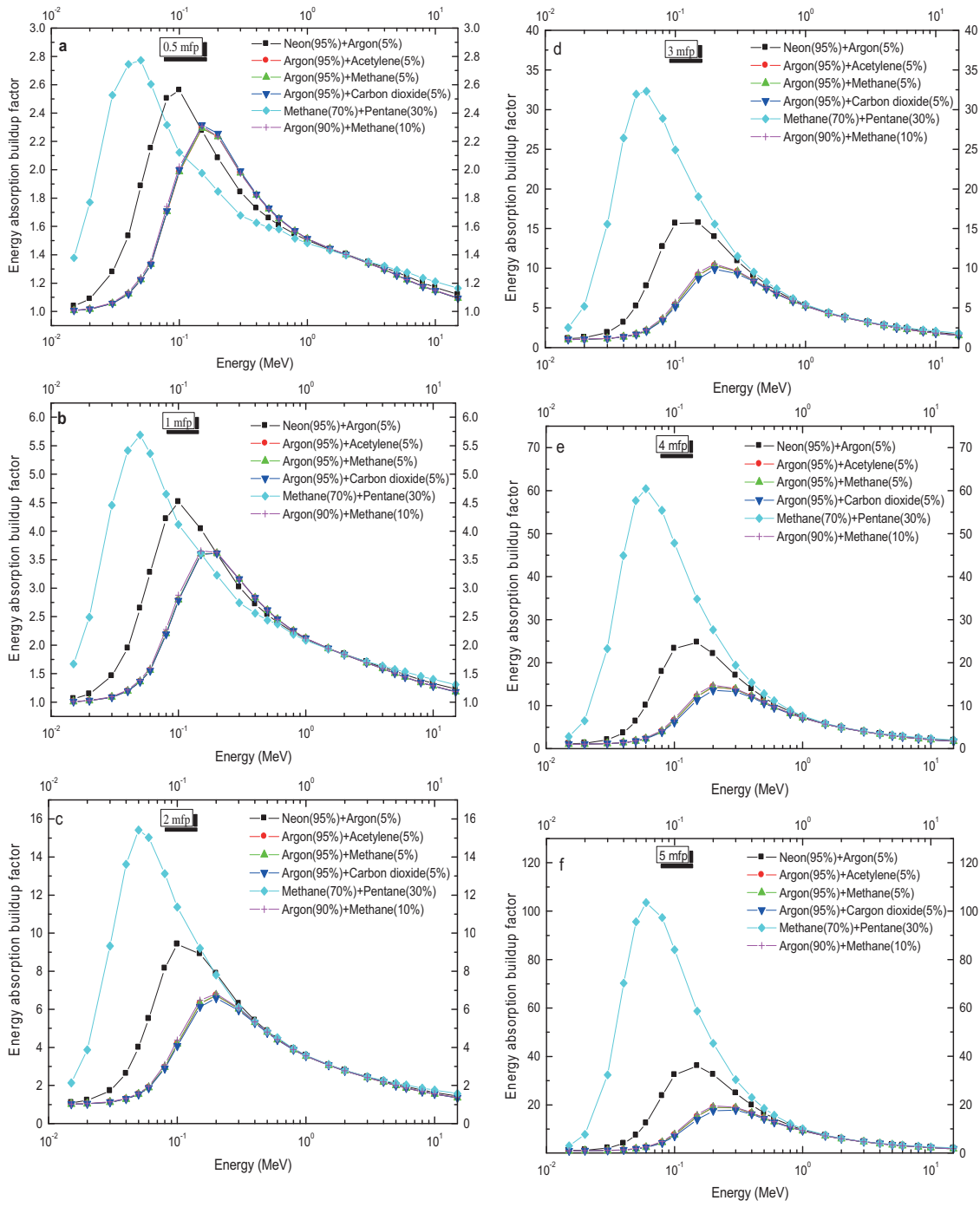

Figure 4 - Chemical compostion effect of gaseous mixture for energy absorption buildup factors; a) 0.5 , b) 1, c) 2, d) 3,e) 4,f) 5, g) 10, h) 15 and i) 20mfp, 

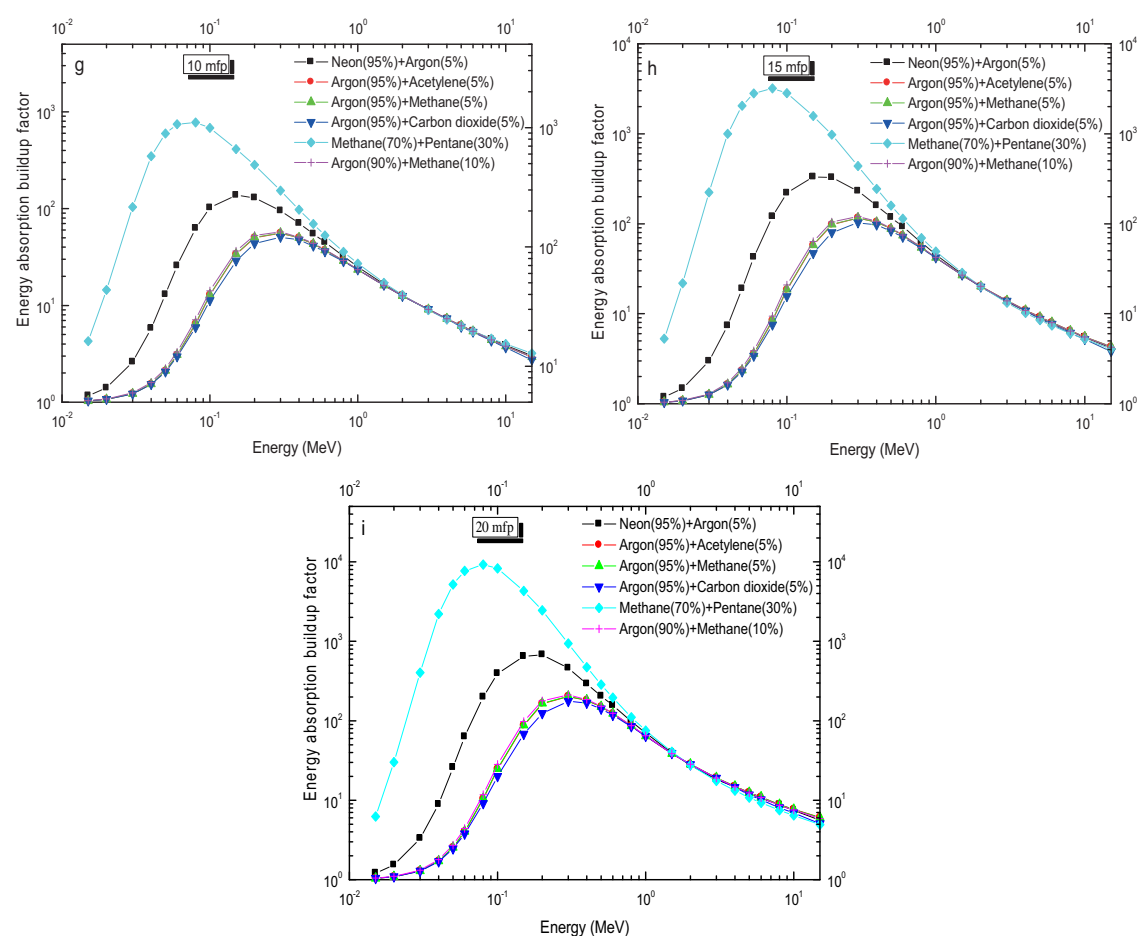

Figure 4 - Continued.

the buildup factors. A very interesting behavior of reverse order of EABF of gaseous mixture is found as photon energy increases beyond $3 \mathrm{MeV}$ and $5 \mathrm{mfp}$ penetration depth. The EABF value increases with increase penetration depth for higher $Z_{e q}$ gaseous mixtures whereas reduces for lower $Z_{e q}$ gaseous mixtures above $15 \mathrm{MeV}$ photon energy. This can be explained by the pair/triplet production in high photon energy which generates secondary radiation after annihilation of rest positions whith electrons. In larger penetration depth of the gaseous mixture ( $\geq 5 \mathrm{mfp}$ ), reverse order EABF is noticeable because of availability of sufficient medium length for interaction.

\section{Conclusions}

- $Z_{e q}$ of the gaseous mixture methane $(70 \%)+$ pentane $(30 \%)$ is found to be 5.2 to 5.3 closer to the $Z_{\text {eff }}$ of tissue $\left(Z_{\text {tissue }}=7.4\right)$ below $100 \mathrm{keV}$ and decreases sharply to 4.2 above a photon energy of $1 \mathrm{MeV}$. 
PHOTON ENERGY ABSORPTION BUILDUP FACTORS OF GASEOUS MIXTURES

- $Z_{e q}$ of neon $(90 \%)+\operatorname{argon}(5 \%)$ is found to be 10.4 to 10.9 for the photon energy range $0.015-15 \mathrm{MeV}$ and other gaseous mixtures have very high $Z_{e q}$.

- Energy absorption buildup factors of the gaseous mixtures are photon energydependent with lower values at low and high photon energies due to photoelectric absorption and pair-production.

- The energy absorption buildup factor slowly increases with the increase in penetration depth and becomes constant afterward.

- For penetration depth of $\leq 5 \mathrm{mfp}$ and photon energy $<3 \mathrm{MeV}$, energy absorption buildup factors are higher for low $Z_{e q}$ gases such as methane (70\%) + pentane (5\%) and Neon (95\%) + argon (5\%).

- As penetration depth increase above $5 \mathrm{mpf}$, energy absorbtion buildup become higher for comparatively hight $Z_{e q}$ gaseous mixtures.

Acknowledgement. The authors would like to acknowledge the technical support of Murat Kurudirek, Department of Physics, Ataturk University.

\section{REFERENCES}

Akinao S. (2002) Calculation of Gamma-Ray Buildup Factors up to depths of $100 \mathrm{mfp}$ by the method of Invariant Embedding, (I) Analysis of accuracy and comparison with other data, Nucl. Sci. Tech. 39, 477-486.

Allard D.J., Nazarali A.M., Chabot C.E. (1992) The N-16 Gamma Radiation Response of GeigerMüller Tubes, Proc. Int. Cong. of the International Radiation Protection Association (IRPA8), 17-22 May, pp. 652-655, Montreal, Canada.

ANSI (1991) American National Standard Institute, Gamma-ray attenuation coefficients and buildup factor for engineering materials, Report ANSI/ANS/6.4.3, American Nuclear Society, La Grange Park, Illinois.

Berger M.J., Hubbell J.H. (1987/1999) XCOM, NIST, Gaithersburg, MD 20, 899, USA.

Chilton A.B., Eisenhauer C.M., Simmons G.L. (1980) Photon point source buildup factors for air, water and iron, Nucl. Sci. Eng. 73, 97-107.

Gelward L., Guilbert N., Jensen K.B., Levring H. (2001) X-ray absorption in matter. Reengineering XCOM, Radiat. Phys. Chem. 60, 23-24.

Gelward L., Guilbert N., Jensen K.B., Levring H. (2004) WinXcom-a program for calculating X-ray attenuation coefficients, Radiat. Phys. Chem. 71, 653-654.

Gopinath D.V., Samthanam K. (1971) Radiation transport in one dimensional finite System-Part I. Development in Anisotropic Source Flux Technique, Nucl. Sci. Eng. 43, 186-196.

Haridas G.N., Nayak M.K., Dev V., Thakkar K.K., Sarkar P.K., Sharma D.N. (2006) Dose build up correction for radiation monitors in high-energy bremsstrahlung photon in radiation fields, Radiat. Prot. Dosim. 118, 233-237.

Harima Y. (1983) An approximation of gamma buildup factors by modified geometrical progression, Nucl. Sci. Eng. 83, 299-309.

Harima Y. (1993) An Historical review and current status of buildup factor calculations and application, Radiat. Phys. Chem. 41, 631-659.

Harima Y., Sakamoto Y., Tanka S., Kawai M. (1986) Validity of geometric progression formula in approximating gamma ray buildup factor, Nucl. Sci. Eng. 94, 24-35. 
Knoll G.F. (2000) Ionization Camber, Radiation Detection and Measurement, 3rd edition, pp. 129-217, John Wiley \& Sons, New York.

Luis A.D. (2009) Update to ANSI/ANS-6.4.3-1991 for low-Z materials and compound materials and review of particle transport theory, UNLV, Las Vegas, NV 89154.

Maron M.J. (1987) Numerical analysis: A Practical approach, Macmillan, New York.

Manohara S.R., Hanagodimath S.M., Gerward L. (2010) Energy absorption buildup factors for thermoluminescent dosimetic materials and their tissue equivalent, Radiat. Phys. Chem. 79, 575-582.

Murat K., Yuksel O. (2011) Energy absorption and exposure buildup factors for some polymers and tissue substitute materials: photon energy, penetration depth and chemical composition dependence, J. Radiol. Prot. 31, 117-128.

Murat K., Bekir D., Metin I., Neslihan E., Yuksel O. (2011) Gamma-ray energy absorption and exposure buildup factor studies in some human tissues with endometriosis, Appl. Radiat. Isotopes 69, 381-388.

Nelson W.R., Hirayama H., Rogers D.W.O. (1985) EGS4 code system, Stanford Linear Accelerator Centre, 265, Stanford, California.

Raza S., Avila R. (2005) Calculation of immersion doses from external exposure to a plume of radioactive material, Health Phys. 89, 247-254.

Sakamoto Y., Tanaka S. (1988) Interpolation of gamma ray buildup factors for point isotropic source with respect to atomic number, Nucl. Sci. Eng. 100, 33-42.

Simmons G.L. (1973) An adjoint gamma-ray moments computer code ADJMOM-I, NBS Technical Note 748, National Bureau of Standards.

Shimizu A. (2002) Calculation of gamma-ray buildup factors upto depth of $100 \mathrm{mfp}$ by method of invariant embedding, (I) analysis of accuracy and comparison with other data, J. Nucl. Sci. Technol. 39, 477.

Shimizu A., Onda T., Sakamoto Y. (2004) Calculation of gamma-ray buildup facor upto depth of $100 \mathrm{mfp}$ by the method of invariant embedding, (III) generation of improved data set, J. Nucl. Sci. Technol. 41, 413-24.

Singh V.P., Badiger N.M. (2011) Study of effective atomic number and electron densities of some gases of radiation detectors, Proc. National Symposium on Nuclear Energy and Health Care (NEHCA-2011), D. Y. Patil University, Kolhapur, India, 22-24 Oct., OP-1, pp. 47.

Singh V.P., Badiger N.M. (2012a) Effective atomic numbers, electron densities and tissue equivalence of some gases and mixtures for dosemetry in radiation detectors, Nucl. Technol. Radiat. Prot. 27 (2), 117-124.

Singh V.P., Badiger N.M. (2012b) Comprehensive study of energy absorption and exposure buildup factor for concrete shielding in photon energy range $0.015-15 \mathrm{MeV}$ upto $40 \mathrm{mfp}$ penetration depth: dependency of density, chemical element, photon energy, Int. J. Nucl. Energy Sci. Technol. 7 (1), 75-99.

Takeuchi K., Tanaka S. (1984) PALLAS-ID (VII). A code for direct integration of transport equation in one-dimensional plane and spherical geometries, JAERI-M 84, 214.

Takeuchi K., Tanaka S. (1985) Point isotropic buildup factor of gamma rays, including bremsstrahlung and annihilation radiation for water, concrete, iron and lead, Nucl. Sci. Eng. 90, 158-164.

Takeuchi K., Tanaka S. (1986) Detailed investigation of the buildup factors and spectra for point isotropic gamma ray sources in vicinity of the k edge in lead, Nucl. Sci. Eng. 93, 376-385. 